\title{
Machine Learning Algorithm-Based Analysis of Efficacy of Pulmonary Surfactant Combined with Mucosolvan in Meconium Aspiration Syndrome of Newborns through Ultrasonic Images
}

\author{
Yanni Ji $\mathbb{D}^{1},{ }^{1}$ Wenqian Lou $(\mathbb{D})^{2}$ and Jianwei Ji $\mathbb{D}^{1}$ \\ ${ }^{1}$ Department of Pediatrics, Yiwu Central Hospital, Yiwu 322000, China \\ ${ }^{2}$ Department of Pediatrics, Yiwu Maternal and Child Health Hospital, Yiwu 322000, China
}

Correspondence should be addressed to Wenqian Lou; 20200120878@nxmu.edu.cn

Received 6 June 2021; Revised 2 July 2021; Accepted 15 July 2021; Published 22 July 2021

Academic Editor: Gustavo Ramirez

Copyright (C) 2021 Yanni Ji et al. This is an open access article distributed under the Creative Commons Attribution License, which permits unrestricted use, distribution, and reproduction in any medium, provided the original work is properly cited.

\begin{abstract}
Objective. The study aimed to explore the efficacy of pulmonary surfactant (PS) combined with Mucosolvan in the diagnosis of meconium aspiration syndrome (MAS) in newborns through ultrasonic images of lung based on machine learning. Methods. 138 cases of infants with MAS were selected as the research subjects and randomly divided into PS group $(n=46)$, Mucosolvan group $(n=46)$, and combination group $(n=46)$. Then, ultrasonic images based on machine learning algorithm were used for examination. On the basis of conventional treatment, the PS group accepted intratracheal PS drip treatment with $100 \mathrm{mg} / \mathrm{kg}$. For the Mucosolvan group, $7.5 \mathrm{mg} / \mathrm{kg}$ of Mucosolvan was added with $50 \mathrm{~g} / \mathrm{L}$ glucose, which was diluted to $3 \mathrm{~mL}$. Then, the mixture was injected intravenously with a micropump for more than $5 \mathrm{~min}$. The combination group received combined treatment of PS and Mucosolvan. If there was no relief or the symptoms aggravated after $12 \mathrm{~h}$ of PS treatment, the patient should be treated again. $7.5 \mathrm{mg} / \mathrm{kg} / \mathrm{d}$ of Mucosolvan was given for 7 days. Mechanical ventilation time, hospitalization time, oxygenation index (OI) before treatment, at $3 \mathrm{~d}$ and at $7 \mathrm{~d}$ after treatment, and arterial/alveolar oxygen ratio $\left(\mathrm{a} / \mathrm{APO}_{2}\right)$ of the three groups were detected and compared. Besides, in-hospital mortality and complication rate of the three groups were statistically compared. Results. Ultrasonic image edge detection based on machine learning algorithm was more condensed and better than Sobel operator. Compared with the PS group and the Mucosolvan group, treatment efficiency, OI at $3 \mathrm{~d}$ and at $7 \mathrm{~d}$ after treatment, and a/APO $\mathrm{O}_{2}$ combination group were increased. Mechanical ventilation time and hospitalization time of the combination group were shortened, and mortality rate of the combination group was reduced $(P<0.05)$. Compared with the situation before treatment, OI at $3 \mathrm{~d}$ and at $7 \mathrm{~d}$ after treatment and $\mathrm{a} / \mathrm{APO}_{2}$ of the combination group were increased, and $\mathrm{OI}$ at $7 \mathrm{~d}$ after treatment and a/APO $\mathrm{A}_{2}$ of the PS group and the Mucosolvan group were increased $(P<0.05)$. Curative effect, mechanical ventilation time, hospitalization time, OI before and after treatment, a/ $\mathrm{APO}_{2}$, and mortality rate during hospitalization of the PS group and the Mucosolvan group had no significant difference $(P>0.05)$. There was no significant difference in the complications rates in the three groups $(P>0.05)$. Conclusion. Pulmonary ultrasound based on machine learning algorithm can be used in the diagnosis of MAS in neonates. PS combined with Mucosolvan is feasible and safe in treating neonatal MAS and effectively improves the pulmonary oxygenation function. Therefore, it is worthy of clinical application.
\end{abstract}

\section{Introduction}

Neonatal MAS is a clinically common neonatal disease, which is a respiratory disease caused by inhalation of meconium contaminants in perinatal children in utero or when delivery. This disease is serious, the mortality rate is high, and the prognosis is poor $[1,2]$. Therefore, timely and effective diagnosis and treatment of MAS in newborns are important to improve neonatal outcomes.

In previous studies, $\mathrm{X}$-rays and clinical manifestations of patients were mostly used for diagnosis and analysis. With the continuous development of medical imaging technology, it has been found that ultrasound imaging can achieve significant effects in the clinical diagnosis of pneumonia, 
atelectasis, and neonatal MAS [3]. Ultrasound imaging technology has the advantages of being noninvasive, convenient, and fast, with dynamic observation. After machine learning algorithms were used for image processing, the image quality and detection accuracy were further optimized and improved [4]. The treatment of MAS is mainly though drug therapy, in which PS and ambroxol hydrochloride (Mucosolvan) can achieve a certain effect when conducting treatment [5, 6]. Zhange et al. [7] combined the use of PS and Mucosolvan to treat newborns with respiratory distress syndrome and found that it helps to improve the immune inflammation, as well as liver and kidney function of those children. Therefore, the combined use of PS and Mucosolvan in the treatment of MAS may achieve good results. There are few reports on the combined use of PS and Mucosolvan in the treatment of MAS, and the efficacy of the combination treatment is unclear and therefore needs to be confirmed by further studies. As a result, in this study, ultrasound imaging of lung based on machine learning algorithm was used to explore the diagnosis of MAS with combination of PS and Mucosolvan, and its effect on efficacy and oxygenation function was observed and safety was analyzed, which aimed to find a safe and effective treatment program of neonatal MAS to improve its treatment level. The specific research methods and results are as follows.

\section{Materials and Methods}

2.1. General Information. 138 children with MAS, hospitalized from June 2016 to June 2019, were selected as the research subjects. The inclusion criteria were as follows: all children had a history of intrauterine distress or choking during labor, and amniotic fluid was contaminated by viscous meconium; shortly after their birth, dyspnea, bruising, or even respiratory failure occurred. Moreover, chest radiography showed granular and flaky shadows on the lungs. The exclusion criteria were as follows: children combined with other congenital defects and infectious diseases and children who had a disorder immune system and a dysfunction in critical organs. All children met the inclusion criteria and no children were excluded. The selected children were randomly divided into three groups according to the envelope grouping method: PS group $(n=46)$, Mucosolvan group $(n=46)$, and combination group $(n=46)$. The test met ethical standards and was approved by the ethics committee of the hospital. Moreover, all the guardians of the children signed the informed consent forms. There was no statistically significant difference in the baseline data of gender, gestational age, birth weight, delivery mode, and $1 \mathrm{~min}$ Apgar score between the three groups $(P>0.05)$, which were comparable, as shown in Table 1.

2.2. Ultrasound Examination. All newborns with MAS were examined with GE Voluson i, and the ultrasonic frequency was set in the range of $10-14 \mathrm{MHz}$. The examination was conducted when the newborns were in a quiet state. The newborn should maintain a supine, lateral, or prone position, with the child's posterior axillary line and anterior axillary line as the boundary. Besides, the lung on each side was divided into front, back, and side areas. It should be noted that the child's ribs were parallel or perpendicular to the ultrasound probe during the inspection. Then, each area of the lungs on both sides was carefully inspected.

2.3. Ultrasound Image Edge Detection Algorithm Based on Machine Learning. The machine learning algorithm used in this study included the four following steps: establishment of ultrasound image training sample set, feature extraction, training algorithm classifier, and simulation test.

The Establishment of Ultrasound Image Training Sample Set. The collected MAS lung ultrasound images of newborns were taken as the experimental data. Among them, 1035 images were selected as the training set. Then, the image automatic annotation algorithm was adopted based on integer discrete cosine transform (DCT) [8] to annotate the images. After that, the annotated images were used as the training samples. The $48 * 48$ image blocks represented positive samples (including valid edge points), and the rest were negative samples. Then, samples were taken with a ratio of $1: 2$ of positive samples to negative samples.

Feature Extraction. The multiscale gradient histogram features and Haar-like features were used to train image samples. The Haar-like feature extracted features through four kinds of rectangles in the block, and the "cumulative sum" method was used for calculation. The calculation equation for the cumulative sum of gray blocks is as follows:

$$
\prod(x, y)=\sum_{x^{\prime} \leq x, y^{\prime} \leq y} G(x, y) .
$$

Here, $\Pi$ is the cumulative sum, $G$ is the gray level, and $(x, y)$ is the coordinate of the image point.

The calculation equation for the number of extracted Haar features is as follows:

$$
\begin{aligned}
& X Y\left(L+1-l \frac{X+1}{2}\right)\left(H+1-h \frac{Y+1}{2}\right), \\
& X=\left[\frac{L}{l}\right], \\
& Y=\left[\frac{H}{h}\right] .
\end{aligned}
$$

Here, $L$ and $H$ are the length and height of $L^{*} H$ image, respectively; $X$ and $Y$ are the maximum horizontal and vertical placement scale factors, respectively.

In this study, the subimage was a $12^{*} 12$ rectangle with $48^{*} 48$ pixels. According to the two, it can be inferred that the number of subimage features was 5776 and the number of Haar feature vectors was $1.73 \times 107$.

Then, multiscale gradient histogram features were used to extract $48^{*} 48$ subimages of training samples. First, the subimage was divided into $3^{*} 3$ image units. Then, the number of gradient directions was determined according to 
TABLE 1: Comparison of baseline data between the three groups.

\begin{tabular}{lcccccc}
\hline Groups & $\begin{array}{c}\text { Number of } \\
\text { cases }\end{array}$ & $\begin{array}{c}\text { Gender } \\
\text { (male/female) }\end{array}$ & $\begin{array}{c}\text { Gestational } \\
\text { age (week) }\end{array}$ & $\begin{array}{c}\text { Birth } \\
\text { weight (kg) }\end{array}$ & $\begin{array}{c}\text { Delivery mode } \\
\text { (cesarean delivery/spontaneous labor) }\end{array}$ & 1 min Apgar score \\
\hline PS group & 46 & $29 / 17$ & $40.21 \pm 1.38$ & $3.11 \pm 0.61$ & $25 / 21$ & $5.26 \pm 1.35$ \\
Mucosolvan group & 46 & $31 / 15$ & $40.09 \pm 1.42$ & $3.06 \pm 0.58$ & $27 / 19$ & $5.18 \pm 1.24$ \\
Combination group & 46 & $28 / 18$ & $40.16 \pm 1.43$ & $3.20 \pm 0.55$ & $28 / 18$ & $5.23 \pm 1.29$ \\
Statistical magnitude & & $\chi^{2}=0.439$ & $F=0.084$ & $F=0.687$ & $\chi^{2}=0.416$ & $>0.039$ \\
$P$ & & $>0.05$ & $>0.05$ & $>0.05$ & $>0.05$ & $>0.05$ \\
\hline
\end{tabular}

actual needs. In the study, the gradient directions were divided into 8 dimensions, so that a $3 * 3 * 8$-dimensional feature vector can be formed. Finally, the number of gradient direction histograms was $2.16 \times 105$.

Training Algorithm Classifier. AdaBoost algorithm [9] was adopted to train the classifier. Then, positive and negative samples were used for experimental testing.

2.4. Treatment Methods. All three groups received conventional treatments such as anti-infection, nutritional support, maintenance of water and electrolytes, and stimulation of respiratory excitement. During the period, attention was paid to controlling the amount of fluid. On the basis of conventional treatment, the PS group, the Mucosolvan group, and the combination group were given $100 \mathrm{mg} / \mathrm{kg}$ PS (poractant alfa injection, Chiesi Farmaceutici S.p.A., dosage form: injection, specification: $1.5 \mathrm{~mL}: 0.12 \mathrm{~g}$, batch number: H20080429) in the trachea for drip therapy, $7.5 \mathrm{mg} / \mathrm{kg}$ Mucosolvan (ambroxol hydrochloride tablets, Boehringer Ingelheim, Shanghai, dosage form: tablets, specification: $30 \mathrm{mg}$, batch number: H20030360) with $50 \mathrm{~g} / \mathrm{L}$ glucose and diluted to $3 \mathrm{ml}$, and then micropumped intravenous injection treatment was taken for more than 5 minutes, and combination treatment of PS and Mucosolvan was conducted. After 12 hours of PS treatment, if there was no remission or aggravation, the treatment should be repeated, while a $1 \mathrm{~kg}$ patient should be injected $7.5 \mathrm{mg}$ Mucosolvan per day for a total of 7 days for treatment.

2.5. Observation Indexes and Detection Methods. After 7 days of treatment, the attending physician evaluated the child's condition and statistically compared the mechanical ventilation time and hospitalization time of the three groups. Blood gas analysis was performed before treatment, 3 days after treatment, and 7 days after treatment; the relevant adoption number was recorded; the oxygenation index (OI) and arterial/alveolar oxygen partial pressure ratio $\left(\mathrm{a} / \mathrm{APO}_{2}\right)$ were calculated, and the mortality and the incidence of complications during hospitalization of the children were statistically compared. Regarding efficacy evaluation [10], curing is that the clinical symptoms disappear after treatment and all the signs indicators return to normal; the marked effect is that the clinical symptoms are significantly improved after treatment and the lesions are significantly reduced or disappear on chest radiography; the effective result is that the clinical symptoms after treatment have improved and the lesions have reduced on chest radiography; and the invalidity is that the clinical symptoms have not improved or even worsened after treatment, and the chest radiography has not seen shrinkage or even complications.

2.6. Statistical Methods. SPSS 19.0 software was used to establish the database and perform statistical analysis of related data. Among them, nonnormal distribution was tested by rank-sum test and count data were tested by chisquare test. Analysis of variance was used for comparison of multiple sets of measurement data, and when $P<0.05$, it was considered statistically significant.

\section{Results}

3.1. Comparison of Ultrasound Image Edge Detection Results Based on Machine Learning Algorithms. In this study, the image edge detection algorithm based on machine learning was used in ultrasonic image processing. Then, the detection results were compared with those of Sobel operator. The simulation test result is shown in Figure 1. Both algorithms can effectively detect edge information (Figures 1(b) and 1(c)). The machine learning edge detection algorithm was more condensed, and the edge of the image was clearer.

3.2. Ultrasound Images. In this study, the main manifestations of lung ultrasound images of newborns with MAS were as follows. All children had lung consolidation with bronchial inflation signs. The consolidation area of severe cases showed large-scale and irregular border features (Figures 2(a) and 2(b)). The mild cases showed small-scale localized consolidation (Figure 2(c)). All children had abnormal pleural line and disappearance of A-line. The main characteristics of abnormal pleural line were the disappearance, thickening, and fuzzification of the pleural line of the lesion (Figures 2(a)-2(d)). Some severe cases had atelectasis (Figure 2(d)). In addition, some cases had pleural effusion (Figure 2(d)).

\subsection{Comparison of Curative Effects in the Three Groups.} Compared with the PS group and the Mucosolvan group, the effective rate of treatment in the combination group increased, and the difference was statistically significant $(P<0.05)$, while the PS group and Mucosolvan group had no statistically significant difference $(P>0.05)$, as shown in Table 2. 


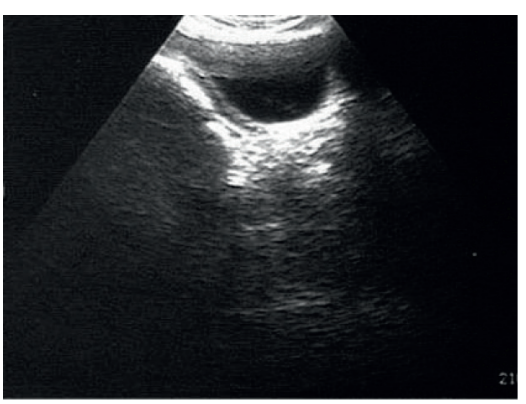

(a)

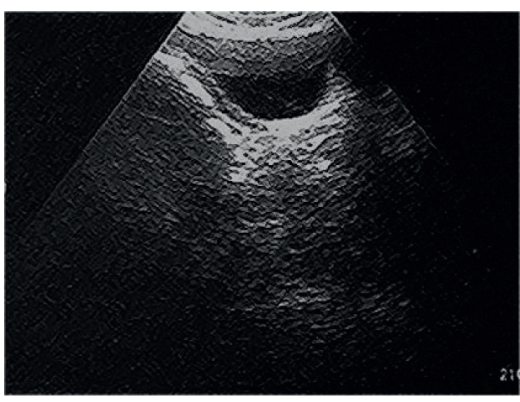

(b)

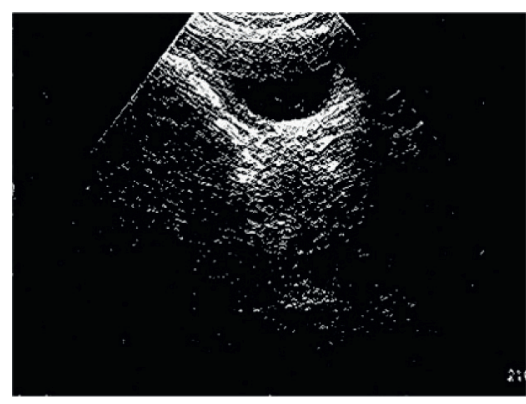

(c)

Figure 1: Detection results of different algorithms. (a) The original image; (b) Sobel operator; (c) machine learning algorithm.

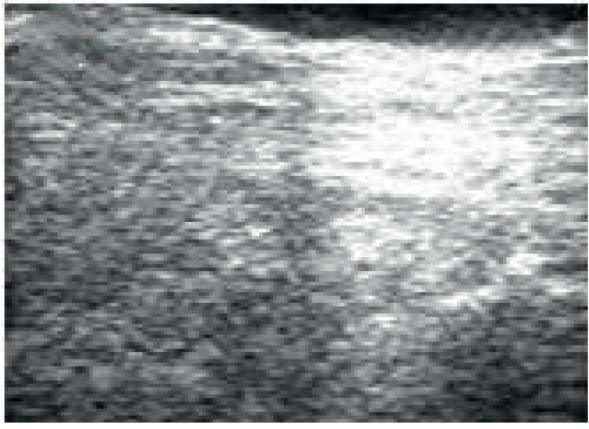

(a)

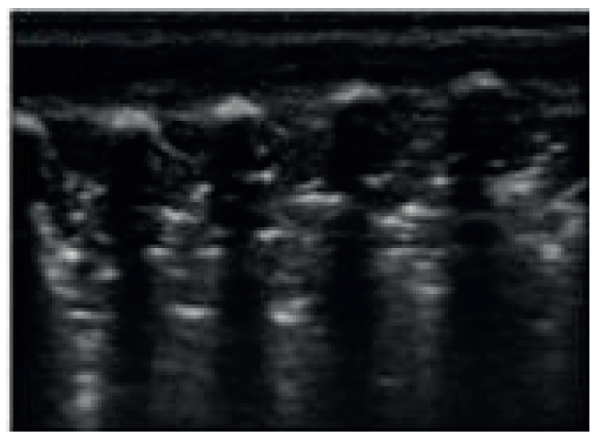

(c)

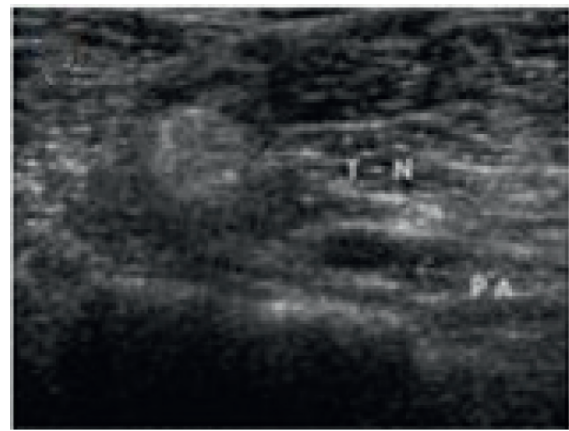

(b)

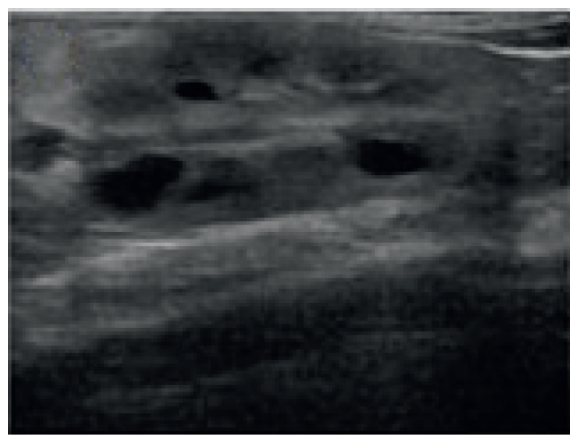

(d)

FIGURE 2: Ultrasonic images of the lungs of newborns with MAS. (a-d) The characteristics of ultrasound images of different patients.

TABLE 2: Comparison of curative effects in the three groups (case (\%)).

\begin{tabular}{lccccc}
\hline Groups & Number of cases & Curing & Marked effect & Effective result & Invalidity \\
\hline PS group & 46 & $14^{\mathrm{ab}}$ & 12 & 13 & 13 \\
Mucosolvan group & 46 & $13^{\mathrm{a}}$ & 14 & 8 & 8 \\
Combination group & 46 & 29 & 8 & 7.652 & 1 \\
$Z$ & & & $<0.05$ & \\
$P$ & & & & \\
\hline
\end{tabular}

${ }^{\mathrm{a}}$ In contrast with the combination group, $P<0.05$; ${ }^{\mathrm{b}}$ in contrast with the Mucosolvan group, $P>0.05$.

3.4. Comparison of Mechanical Ventilation Time, Hospitalization Time, and Mortality during Hospitalization in the Three Groups. Compared with the PS group and the Mucosolvan group, the mechanical ventilation time and hospitalization time of the combination group were shortened, and the mortality during hospitalization was reduced. The difference was statistically significant $(P<0.05)$. There was no statistically significant difference in the mechanical ventilation 
time, hospitalization time, and mortality during hospitalization between the PS group and the Mucosolvan group $(P>0.05)$, as shown in Table 3.

3.5. Comparison of Blood Gas Analysis in the Three Groups before and after Treatment. Compared with the PS group and the Mucosolvan group, the OI and $\mathrm{a} / \mathrm{APO}_{2}$ of the combination group were increased after 3 days and 7 days, and the difference was statistically significant $(P<0.05)$. The OI and a/ $/ \mathrm{APO}_{2}$ values of the combination group were increased after 3 days and 7 days compared with the values before treatment; and the OI and a/ $\mathrm{APO}_{2}$ values of the PS group and the Mucosolvan group were also increased, and the difference was statistically significant $(P<0.05)$. There was no significant difference in OI and a/APO2 values between the PS group and the Mucosolvan group before and after treatment $(P>0.05)$, as shown in Table 4 .

3.6. Comparison of the Incidence of Complications in the Three Groups. There was no significant difference in the incidence of complications such as pulmonary infection, intracranial hemorrhage, pneumorrhagia, and respiratory failure among the three groups $(P>0.05)$, as shown in Table 5 .

\section{Discussion}

MAS, or meconium aspiration pneumonia, is a common aspiration pneumonia common in full-term and overdue babies before or during delivery. It is caused by the inhalation of meconium contaminants in the perinatal child in utero or during delivery, causing the child to have an inflammatory response to infection, causing damage to pulmonary vascular endothelial cells and alveolar epithelial cells, resulting in reduced synthesis of PS, resulting in alveolar atrophy and hyaline membrane disease; the mechanical obstruction of the small airway can cause uneven warming of the lungs, emphysema, atelectasis, interstitial lung edema, and so forth, which can cause significant lung damage. Once the lung compliance is reduced, the child will have difficulty in ventilation, which may further lead to the occurrence of serious complications such as severe hypoxia, hypercapnia, acute respiratory distress syndrome, pulmonary hypertension, respiratory failure, and other threats to children's life [11-13]. In recent years, with the continuous development of medical technology, the diagnosis and treatment of neonatal diseases such as MAS have made great progress, but its efficacy and prognosis still need to be further improved. It is also focused on the efficacy and prognosis of neonatal MAS in this study. The results of this research showed that, after treatment of neonatal MAS, a considerable number of children had poor treatment effects, and some children even had serious adverse prognoses such as death. Due to the poor efficacy and prognosis of neonatal MAS, improving the efficacy and prognosis of neonatal MAS is a problem that needs to be solved urgently.

The treatment of MAS is mainly through drug therapy, of which PS is the more commonly used drug [14]. PS drugs are synthetic preparations, which can effectively compensate for the related diseases of the body's lack of lung surfactant, improve the alveolar surface tension and lung ventilation, and improve the compliance of the lung. Its application effect in the treatment of MAS has been affirmed by multiple studies $[15,16]$. Ambroxol hydrochloride (Mucosolvan) is also one of the treatment drugs for MAS, which can quickly promote sputum excretion and promote lung maturation and can accelerate the synthesis and secretion of PS by stimulating the development of alveolar type II cells. In this study, they were combined in the treatment of MAS. It was found that, compared with the PS group and the Mucosolvan group, the effective rate of the combination group was increased, and the difference was statistically significant $(P<0.05)$. Compared with the PS group and the Mucosolvan group, the OI and a/ $\mathrm{APO}_{2}$ were increased in the combination group $3 \mathrm{~d}$ and $7 \mathrm{~d}$ after treatments, and the difference was statistically significant $(P<0.05)$. This was consistent with the research results of Natarajan et al. [17]. Besides, it was found that, compared with the PS group and the Mucosolvan group, the duration of mechanical ventilation and hospital stay in the combination group were shortened, and the mortality during hospital stay was reduced, with statistically significant differences $(P<0.05)$. This was consistent with the conclusion of El Shahed et al. [18].

In this study, machine learning algorithm was applied to medical ultrasonic image processing. It was found that, compared with Sobel operator, the ultrasonic image detection effect was the best based on machine learning algorithm. It was consistent with the research results of Chan et al. [19], indicating that the machine learning algorithm can increase the clarity of ultrasonic image edge detection, with better retention, thus improving the image quality. This study was to observe the efficacy, oxygenation, and safety of pulmonary surfactant combined with Mucosolvan in the treatment of MAS. The results of this study showed that the combination of PS and Mucosolvan for neonatal MAS can improve the effectiveness of combined treatment of neonatal MAS, the OI and a/ $\mathrm{APO}_{2}$ values were also higher 3 days and 7 days after treatments, and the oxygenation function of the child was significantly improved; meanwhile the mechanical ventilation time and hospital stay of the child were shortened, the mortality rate was reduced, and the efficacy and prognosis were significantly improved. Regardless of the method adopted to treat neonatal MAS, there were no significant differences in the incidence of complications such as pulmonary infection, intracranial hemorrhage, pneumorrhagia, and respiratory failure. The combination of PS and Mucosolvan was good for the treatment of neonatal MAS, and it is a safe and effective treatment for neonatal MAS. The mechanism may be as follows: the exogenous PS and Mucosolvan promote the synthesis and secretion of PS, which can double guarantee the PS required to maintain normal lung function, and rapidly improve the clinical symptoms and pulmonary oxygenation dysfunction caused by disease; moreover Mucosolvan promotes sputum excretion function, which can further promote the elimination of foreign bodies, minimize the inflammation and related damage to the lungs, and promote the recovery of lung function. Also, it can promote neonatal lung maturity, the 
TABLE 3: Comparison of mechanical ventilation time and hospitalization time and mortality during hospitalization in the three groups.

\begin{tabular}{lcccc}
\hline Groups & Number of cases & Mechanical ventilation time $(\mathrm{d})$ & Hospitalization time $(\mathrm{d})$ & Mortality $($ case $(10 \%))$ \\
\hline PS group & 46 & $11.25 \pm .92^{\mathrm{ab}}$ & $27.88 \pm 4.56^{\mathrm{ab}}$ & $8(17.39)^{\mathrm{ab}}$ \\
Mucosolvan group & 46 & $10.18 \pm 2.75^{\mathrm{a}}$ & $26.95 \pm 4.22^{\mathrm{a}}$ & $7(15.22)^{\mathrm{a}}$ \\
Combination group & 46 & $3.96 \pm 0.58$ & $18.62 \pm 3.35$ & $0(0.00)$ \\
Statistical magnitude & & $F=130.194$ & $F=72.013$ & $<2.527$ \\
$P$ & & $<0.05$ & $<0.05$ & $<0.05$ \\
\hline
\end{tabular}

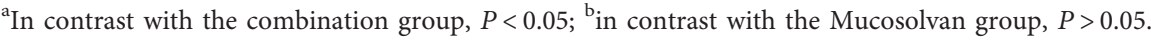

TABLE 4: Comparison of blood gas analysis in the three groups before and after treatment.

\begin{tabular}{|c|c|c|c|c|c|c|c|}
\hline \multirow{2}{*}{ Groups } & \multirow{2}{*}{ Number of cases } & \multicolumn{2}{|c|}{ Prior treatment } & \multicolumn{2}{|c|}{3 days after treatment } & \multicolumn{2}{|c|}{7 days after treatment } \\
\hline & & OI & $\mathrm{a} / \mathrm{APO}_{2}$ & OI & $\mathrm{a} / \mathrm{APO}_{2}$ & OI & $\mathrm{a} / \mathrm{APO}_{2}$ \\
\hline PS group & 46 & $128.78 \pm 23.56$ & $0.12 \pm 0.05$ & $133.78 \pm 24.16^{\mathrm{ac}}$ & $0.14 \pm 0.06^{\mathrm{ac}}$ & $136.65 \pm 28.78^{\mathrm{ac}}$ & $0.15 \pm 0.08^{\mathrm{ac}}$ \\
\hline Mucosolvan group & 46 & $125.98 \pm 22.42$ & $0.13 \pm 0.05$ & $129.62 \pm 23.68^{\text {be }}$ & $0.15 \pm 0.06^{\text {be }}$ & $133.15 \pm 25.74^{\text {be }}$ & $0.16 \pm 0.08^{\text {be }}$ \\
\hline Combination group & 46 & $126.63 \pm 23.55$ & $0.12 \pm 0.04$ & $175.56 \pm 26.62^{\mathrm{d}}$ & $0.26 \pm 0.07^{\mathrm{d}}$ & $251.44 \pm 29.82^{\mathrm{d}}$ & $0.35 \pm 0.06^{\mathrm{d}}$ \\
\hline$F$ & & 0.184 & 0.697 & 48.075 & 50.562 & 262.671 & 106.866 \\
\hline$P$ & & $>0.05$ & $>0.05$ & $<0.05$ & $<0.05$ & $<0.05$ & $<0.05$ \\
\hline
\end{tabular}

Compared with the combination group, ${ }^{\text {a }} P<0.05$ and ${ }^{\mathrm{b}} P<0.05$, while compared with the Mucosolvan group, ${ }^{\mathrm{c}} P>0.05$. Compared with the same group before treatment, ${ }^{\mathrm{d}} P<0.05,{ }^{\mathrm{e}} P>0.05$, and ${ }^{\mathrm{f}} P>0.05$.

TABLE 5: Comparison of the incidence of complications in the three groups.

\begin{tabular}{|c|c|c|c|c|c|c|}
\hline Groups & Number of cases & $\begin{array}{c}\text { Pulmonary } \\
\text { infection (cases) }\end{array}$ & $\begin{array}{c}\text { Intracranial } \\
\text { hemorrhage (case) }\end{array}$ & $\begin{array}{l}\text { Pneumorrhagia } \\
\text { (cases) }\end{array}$ & $\begin{array}{c}\text { Respiratory } \\
\text { failure (cases) }\end{array}$ & $\begin{array}{c}\text { The overall } \\
\text { incidence (cases (\%)) }\end{array}$ \\
\hline PS group & 46 & 3 & 0 & 1 & 1 & $5(10.86)$ \\
\hline Mucosolvan group & 46 & 2 & 1 & 1 & 2 & $6(13.04)$ \\
\hline Combination group & 46 & 2 & 0 & 0 & 1 & $3(6.52)$ \\
\hline$\chi^{2}$ & & & & & & 1.113 \\
\hline$P$ & & & & & & $>0.05$ \\
\hline
\end{tabular}

further recovery of lung function in children, and the treatment effect and reduce the occurrence of adverse prognosis $[17,20]$. However, the sample size of this study was small, and the treatment effect and test results might be affected by the operator's skill level and surrounding environment; as a result, in order to clarify the effect of PS combined with Mucosolvan in neonatal MAS, a larger sample size is needed for further comprehensive researches and confirmation.

In summary, pulmonary ultrasound based on machine learning algorithm can be used in the diagnosis of MAS in newborns. PS combined with Mucosolvan is a safe and feasible treatment method for neonatal MAS.

\section{Data Availability}

The data used to support the findings of this study are available from the corresponding author upon request.

\section{Conflicts of Interest}

The authors declare no conflicts of interest.

\section{References}

[1] M. Choudhary, M. K. Meena, N. Chhangani, D. Sharma, J. S. Choudhary, and S. K. Choudhary, "To study prevalence of persistent pulmonary hypertension in newborn with meconium aspiration syndrome in western Rajasthan, India: a prospective observational study," Journal of Maternal-Fetal and Neonatal Medicine, vol. 29, no. 2, pp. 324-327, 2016.

[2] J. Ma, X. L. Wang, Z. Y. Li, and T. Y. Chen, "Effect of PS combined with mucosolvan on immune function, liver and kidney function in neonatal respiratory distress syndrome," Journal of Hainan Medical University, vol. 22, no. 20, pp. 141-145, 2016.

[3] M. Hiles, A.-M. Culpan, C. Watts, T. Munyombwe, and S. Wolstenhulme, "Neonatal respiratory distress syndrome: chest X-ray or lung ultrasound? A systematic review," Ultrasound, vol. 25, no. 2, pp. 80-91, 2017.

[4] B. J. Erickson, P. Korfiatis, Z. Akkus, and T. L. Kline, "Machine learning for medical imaging," RadioGraphics, vol. 37, no. 2, pp. 505-515, 2017.

[5] Y. Zhang, Z. Y. Han, and F. Peng, "Progress in pharmacological and clinical research of ambroxol in the treatment of children's respiratory diseases," World Latest Medicine Information, vol. 34, p. 28, 2017.

[6] Y. Zhou and X. G. Zhou, "Clinical types and characteristics of the MAS in newborns," Chinese Journal of Contemporary Pediatrics, vol. 5, 2000.

[7] S. L. Zhang, Y. J. Hou, B. X. Huang, G. P. Xu et al., "Budesonide suspension associated with ambroxol hydrochloride to treat MAS of newborn by atomizing inhalation with oxygen-driven," Journal of Xinxiang Medical College, vol. 2, 2010.

[8] D. Raimondi, G. Orlando, Y. Moreau, and W. F. Vranken, "Ultra-fast global homology detection with discrete cosine transform and dynamic time warping," Bioinformatics, vol. 34, no. 18, pp. 3118-3125, 2018. 
[9] K. Li, G. Zhou, J. Zhai, F. Li, and M. Shao, "Improved PSO_ adaboost ensemble algorithm for imbalanced data," Sensors, vol. 19, no. 6, Article ID 1476, 2019.

[10] L. X. Hao and F. Wang, "Effectiveness of high-frequency oscillatory ventilation for the treatment of neonatal MAS," Medicine, vol. 98, no. 43, 2019.

[11] D. Louis, V. Sundaram, K. Mukhopadhyay, S. Dutta, and P. Kumar, "Predictors of mortality in neonates with meconium aspiration syndrome," Indian Pediatrics, vol. 51, no. 8, pp. 637-640, 2014.

[12] J. Lee, R. Romero, K. A. Lee et al., "MAS: a role for fetal systemic inflammation," American Journal of Obstetrics and Gynecology, vol. 214, no. 3, pp. 366-e1, 2019.

[13] P. H. Haakonsen Lindenskov, A. Castellheim, O. D. Saugstad, and T. E. Mollnes, "Meconium aspiration syndrome: possible pathophysiological mechanisms and future potential therapies," Neonatology, vol. 107, no. 3, pp. 225-230, 2015.

[14] A. I. El Shahed, P. A. Dargaville, A. Ohlsson, and R. Soll, "Surfactant for MAS in term and late preterm infants," Cochrane Database of Systematic Reviews, vol. 2014, no. 12, Article ID CD002054, 2014.

[15] J. Liu, H.-Y. Cao, and W. Fu, "Lung ultrasonography to diagnose meconium aspiration syndrome of the newborn," Journal of International Medical Research, vol. 44, no. 6, pp. 1534-1542, 2016.

[16] K. S. Rana, M. C. Konar, K. Islam, K. L. Barik, K. Nayek, and A. K. Datta, "Study on effects of steroid on clinical course, short-term and long-term outcomes in neonates with meconium aspiration syndrome," Journal of Neonatal Nursing, vol. 24, no. 5, pp. 257-260, 2018.

[17] C. K. Natarajan, M. J. Sankar, K. Jain, R. Agarwal, and V. K. Paul, "Surfactant therapy and antibiotics in neonates with meconium aspiration syndrome: a systematic review and meta-analysis," Journal of Perinatology, vol. 36, no. Suppl 1, pp. S49-S54, 2016.

[18] A. I. El Shahed, P. A. Dargaville, A. Ohlsson, and R. Soll, "Surfactant for meconium aspiration syndrome in term and late preterm infants," Cochrane Database of Systematic Reviews, vol. 2014, no. 12, Article ID CD002054, 2014.

[19] Y. H. Chan, Y. Z. Zeng, H. C. Wu, M. C. Wu, and H. M. Sun, "Effective pneumothorax detection for chest X-ray images using local binary pattern and support vector machine," Journal of healthcare engineering, vol. 2018, Article ID 2908517, 2018.

[20] F. Yang, "Oxygen-driving and atomized mucosolvan inhalation combined with holistic nursing in the treatment of children severe bronchial pneumonia," Pakistan Journal of Pharmaceutical Sciences, vol. 28, no. 4 Suppl, pp. 1477-1480, 2015. 